\title{
DISCURSO Y PODER EN ECUADOR: ANÁLISIS DEL DISCURSO DE LOS PRESIDENTES RAFAEL CORREA DELGADO Y LENIN MORENO GARCÉS
}

\section{SPEECH AND POWER IN ECUADOR: SPEECH ANALYSIS OF THE PRESIDENTS RAFAEL CORREA DELGADO AND LENIN MORENO GARCÉS}

Gladiz Salazar-Atiencie

Universidad Técnica Particular de Loja, Ecuador

(iD) https://orcid.org/0000-0002-8224-7239

María Isabel Punín Larrea

Universidad Técnica Particular de Loja, Ecuador

(iD https://orcid.org/0000-0001-5052-824X

Autor para correspondencia: Gladiz Salazar-Atiencie, email: gasalazar3@utpl.edu.ec

Resumen

Ecuador desde el 2007 y hasta el 24 de mayo de 2021, estará gobernado por un proyecto político denominado: La Revolución Ciudadana (Alianza País-Listas 35), partido que ha sufrido una serie de discrepancias y el distanciamiento del presidente L. Moreno. Este artículo usa los postulados del Análisis del Discurso, relacionados con las estructuras semánticas, compuestas de complejidad léxica, uso de superlativos y tendencias ideológicas para analizar los mensajes emitidos por Rafael Correa Delgado (2006-2009-2013) y Lenin Moreno Garcés (2017), considerando tres momentos: 1. Campaña electoral, 2. Plan de gobierno y 3. Toma del poder. Sistematiza las promesas usadas en el discurso según los tres momentos mencionados, para luego analizar de manera cronológica y cualitativa las ofertas que lograron cristalizarse durante el ejercicio del poder presidencial. Existe un cambio progresivo del discurso público, debido a dos variantes, la primera asegurar la permanencia en el poder y la segunda, justificar la situación de crisis del país, pero en cualquiera de los casos siempre está conectada con el discurso populista. En el discurso de Rafael Correa se identifica una estrategia definida ideológicamente. Mientras que Lenin Moreno tiende a improvisar, usando un discurso variable, sin conceptos concretos, ni estratégicos.

Palabras claves: democracia, discurso, ideología, poder, Ecuador.

\begin{abstract}
From 2007 until May 24th, 2021, Ecuador will be governed by a political project called: La Revolución Ciudadana (Alianza Pais-Listas 35), a political party that has suffered a series of discrepancies and the distancing of
\end{abstract}

Global Media Journal México, 18(34), 70-93, enero - junio 2021. 
President L. Moreno. This article takes the postulates of Discourse Analysis related to semantic structures, composed of lexical complexity, use of superlatives and ideological tendencies to analyze the messages issued by Rafael Correa D. (2006-2009-2013) and Lenin Moreno G. (2017), considering three moments of study: electoral campaign, government plan and seizure of power. It systematizes the presidential promises used in the speech according to the three mentioned moments, to later analyze chronologically and qualitatively the offers that managed to crystallize during the presidential power exercise. There is a progressive change in public discourse due to two variants, the first to ensure permanence in power and the second to justify the crisis in the country, but in any of the cases, it is always connected with the populist discourse. In Rafael Correa's speech, an ideologically defined strategy is identified. Meanwhile Lenin Moreno, tends to improvise, having a interchangeable discourse without well-defined or strategic concepts.

Keywords: democracy, ideology, speech, power, Ecuador.

Recibido: 23/02/2021

Aceptado: 27/05/2021

\section{Introducción}

La Real Academia Española (RAE), define al discurso como la "facultad racional con que se infieren unas cosas de otras", además, destaca que es una "serie de las palabras y frases empleadas para manifestar lo que se piensa o se siente" (RAE, 2001) que los políticos usan con frecuencia como una forma de llegar a su electorado, considerando además que la política es parte de una esfera social marcada por prácticas discursivas (Van Dijk, 2005).

Michel Foucault, entendía que el discurso se da a partir de los "conocimientos socialmente construidos de algún aspecto de la realidad sociopolítica" (Fair, 2010), es decir, es una construcción social que permite conocer, en cierto modo, las intenciones de quien lo emite, de allí la importancia del discurso político durante la campaña electoral y en el ejercicio del poder, catalogadas como unidades de análisis del presente artículo.

En otras palabras, el discurso como "práctica política" (Chilton y Shäffner, 2002; Wodak y Menz, 1990) y como tal, parte de una pieza ideológica tomada de la realidad para medir de manera subjetiva la coherencia de los gobernantes en cuestión.

Teun Van Dijk (2003) se refiere a componentes principales que definen al discurso como: el significado, la forma, dimensión social y este a su vez tiene la acción y la interacción. Al ser el discurso una expresión comunicativa, fácil y cada vez con mayor impulso, es utilizada en el campo político, por tanto, es necesario identificar cuál es esa relación entre la comunicación y el discurso político.

Virno (citado por Bermúdez y Choi, 2014) se refiere a esta relación como indivisible, así: 
...las dos célebres definiciones aristotélicas, el hombre como "animal con lenguaje” y como “animal político”, no son, según Virno, complementarias, son en realidad "coextensivas, indiscernibles, lógicamente equivalentes” (Virno, 2005: 58). La discusión a dar no es sobre los usos políticos del discurso, sino sobre la politicidad intrínseca del lenguaje. Lejos de ser un tipo especifico de actividad discursiva determinada por una esfera de lo social, la política es en realidad, consubstancial al lenguaje, ambas son fundamentalmente praxis (p. 103).

Esta conexión del discurso y la política no es ajena al contexto ecuatoriano, dicha habilidad es utilizada por los actores políticos de forma oral, así como en el discurso publicitario, y es recurrente en los procesos de campaña electoral para lograr la mayoría de los votos durante los comicios, usados incluso en los procesos de dictaduras blandas que vivió el país en la década de los setenta y con el regreso de la democracia.

Alianza PAÍS, es un movimiento político fundado el 3 de abril de 2006 (Movimiento Alianza PAIS, 2017), que llevó a la presidencia a Rafael Correa Delgado (1963) y a Lenin Moreno Garcés (1953) a la vicepresidencia para el periodo 2007-

\footnotetext{
${ }^{1}$ La página del Consejo Nacional Electoral no tiene disponible este informe, por lo que se tomó los datos generados por los observadores de la OEA en su lugar. ${ }^{2}$ Algunos casos de corrupción que han llegado a las cortes de la justicia ecuatoriana, y que han sido enfrentados por funcionarios y allegados al "correísmo" son: caso
}

2011, con 3517635 votos en segunda vuelta (Consejo Nacional Electoral, 2006, p. 236).

En el año 2008, entra en vigor la nueva Constitución y junto a ella el régimen de transición que disponía un nuevo proceso electoral para la presidencia. Rafael Correa y Lenin Moreno vuelven a las urnas para el periodo 2009-2013, ganando en primera vuelta la reelección, con un total de 3,584,236 votos ciudadanos ${ }^{1}$ (Organización de Estados Americanos, 2010 p. 21).

Para el periodo 2013-2017, Rafael Correa se postula como candidato por tercera vez a la presidencia de Ecuador y Jorge Glas Espinel para la vicepresidencia. Este binomio obtiene 4,918,482 votos y logra nuevamente ganar en la primera vuelta electoral (Consejo Nacional Electoral, 2013, p. 14).

De forma consecutiva, desde el año 2007 hasta el 2017, la mayoría de la población electoral ecuatoriana prefirió votar por la llamada: "Revolución Ciudadana", bandera oficial de Alianza PAÍS. Para el periodo 2017-2021, el gobierno de Lenin Moreno inició su presidencia con 5062018 votos a favor en segunda vuelta, mientras que Jorge Glas Espinel fue electo como vicepresidente (Consejo Nacional Electoral, 2017, p. 9).

No obstante, ya en campaña, Lenin Moreno evidencia una ruptura política, y ofreció ser intransigente con los casos de corrupción en proceso (Constante, 2017)2. El 3 de agosto de 2017, Lenin

sobornos 2012-2016, llamado también "arroz verde"; caso Petroecuador (transferencias de dinero a cuentas de compañías offshore); aportes ilegales a las campañas de Alianza PAIIS; caso Gabela (compra de helicópteros Dhruv); caso Petro-China (entrega excesiva de petróleo ecuatoriano); caso Refinería del Pacífico (sobreprecios). 
Moreno deja sin funciones a J. Glas, a través del Decreto Ejecutivo $\mathrm{N}^{\circ} 100$, y el 28 de octubre del mismo año, la Contraloría General del Estado notificó la destitución del vicepresidente de la República (Diario El Comercio, 2017).

Este artículo busca analizar los mensajes promocionales de las tres campañas presidenciales de Rafael Correa Delgado (2006-2009-2013) y a quien desde aquí en adelante se citará con las iniciales R.C., y la campaña de Lenin Moreno Garcés (2017) con sus iniciales L.M.

Se consideró importante iniciar con la caracterización de los dos personajes principales, esto como parte del cuadro ideológico de los políticos analizados, destacando varias coincidencias, de las cuales, resaltamos su condición presidencial, el partido político y su elección en el marco de votaciones democráticas simples.

La muestra o unidad de estudio está compuesta por los mensajes distribuidos durante la publicidad de las campañas electorales (Tabla 1). Los escritos en el plan de gobierno y el discurso oral proclamado al inicio de la gestión se han obtenido a través de la web, en la página oficial del Consejo Nacional Electoral y en las páginas de medios de comunicación nacionales e internacionales, como parte del análisis contextual de la muestra en estudio.

El corpus de estudio se centra en el análisis del discurso de tres momentos y de los dos niveles de compromiso (posibles electores y potenciales votantes) que asumieron R.C. y L.M., considerando los mensajes cortos usados de manera reiterativa.

\section{Tabla 1}

Tres frases utilizadas en campaña por el expresidente Rafael Correa y el presidente Lenin Moreno

\begin{tabular}{|c|c|c|}
\hline Campaña & Año & Frases \\
\hline \multirow{9}{*}{$\begin{array}{l}\text { Rafael } \\
\text { Correa }\end{array}$} & \multirow{3}{*}{2006} & Dale, Correa \\
\hline & & La patria vuelve \\
\hline & & No a la partidocracia \\
\hline & \multirow{3}{*}{2009} & Dale, patria \\
\hline & & Patria altiva y soberana \\
\hline & & La revolución ciudadana está en marcha y nada la detiene \\
\hline & \multirow{3}{*}{2013} & Ya tenemos presidente, tenemos a Rafael \\
\hline & & Hasta la victoria siempre \\
\hline & & Patria para siempre \\
\hline \multirow{3}{*}{$\begin{array}{l}\text { Lenin } \\
\text { Moreno }\end{array}$} & \multirow{3}{*}{2017} & Vamos por más \\
\hline & & Unidos somos invencibles \\
\hline & & El futuro no se detiene \\
\hline
\end{tabular}

Global Media Journal México, 18(34), 70-93, enero - junio 2021. 


\section{Líneas argumentales destacadas del Plan de Gobierno}

El artículo 97 del Código de la Democracia (2018) establece como requisito obligatorio para los candidatos a la presidencia de la República y a todos los aspirantes a cargos gubernamentales presentar sus planes de gobierno, de ese modo, la ciudadanía tendrá la posibilidad de conocer las ofertas electorales y decidir a quién otorgarle su voto. Para el propósito de este trabajo, se leyeron los cuatro planes de gobierno presentados por R.C. en 2007 y 2013 y por L.M. en 2017; de estos se ha extraído las palabras y frases más repetidas en los textos que tenían relación directa con los eslóganes de las campañas electorales, y con los discursos orales que los presidentes electos declamaron el día de su investidura presidencial (Tabla 2).

\section{Tabla 2}

Argumentos de los Planes de Gobierno por el expresidente Rafael Correa y el presidente Lenin Moreno

\begin{tabular}{|c|c|c|}
\hline Campaña & Año & Argumentos \\
\hline \multirow{11}{*}{$\begin{array}{l}\text { Rafael } \\
\text { Correa }\end{array}$} & \multirow{7}{*}{2007} & Revolución Ciudadana \\
\hline & & $\begin{array}{l}\text { Dale trabajo, crédito y trabajo digno, vivienda, cuidado al ambiente, } \\
\text { educación, salud, desarrollo }\end{array}$ \\
\hline & & Patria nueva \\
\hline & & Para volver a tener Patria \\
\hline & & Cambio radical \\
\hline & & Sepultar a la partidocracia \\
\hline & & Renovación de los actores políticos \\
\hline & 2009 & No se encuentra información \\
\hline & \multirow{3}{*}{2013} & Revolución Ciudadana \\
\hline & & Socialismo del "Buen vivir" \\
\hline & & Patria de todos y de todas \\
\hline \multirow{6}{*}{$\begin{array}{l}\text { Lenin } \\
\text { Moreno }\end{array}$} & \multirow{6}{*}{2017} & $\begin{array}{l}\text { Revolución Ciudadana. Revolución política, ética, económica, } \\
\text { productiva y del trabajo, rural, social, ecológica, del conocimiento y las } \\
\text { capacidades, cultural, de la justicia, seguridad y convivencia, urbana y } \\
\text { de los territorios, de la soberanía y la integración, de la juventud }\end{array}$ \\
\hline & & La década ganada \\
\hline & & Patria grande \\
\hline & & Primero Ecuador \\
\hline & & El pasado no volverá \\
\hline & & Socialismo del Buen Vivir \\
\hline
\end{tabular}

Nota. Elaborado a partir de los Planes de Gobierno de 2007, 2013 y 2017.

Global Media Journal México, 18(34), 70-93, enero - junio 2021. 


\section{Tabla 3}

Argumentos principales en los discursos de toma de posesión por el expresidente Rafael Correa y el presidente Lenin Moreno

\begin{tabular}{|c|c|c|}
\hline Campaña & Año & Argumentos \\
\hline \multirow{3}{*}{$\begin{array}{l}\text { Rafael } \\
\text { Correa }\end{array}$} & 2007 & $\begin{array}{l}\text { "Ejes de la revolución ciudadana: } \\
\text { 1. Revolución constitucional } \\
\text { 2. Lucha contra la corrupción } \\
\text { 3. Revolución económica } \\
\text { 4. Revolución de la educación y la salud } \\
\text { 5. Revolución por la integración latinoamericana. } \\
\text { Cambio radical y profundo } \\
\text { Grupos postergados } \\
\text { La patria vuelve } \\
\text { La patria ya es de todos }\end{array}$ \\
\hline & 2009 & $\begin{array}{l}\text { La revolución ciudadana es irreversible } \\
\text { Larga y triste noche neoliberal } \\
\text { Revolución de los oprimidos } \\
\text { Patria } \\
\text { Patria nueva } \\
\text { Prensa corrupta } \\
\text { Gente de manos limpias y corazones ardientes } \\
\text { Socialismo del siglo XXI } \\
\text { Vende patrias }\end{array}$ \\
\hline & 2013 & $\begin{array}{l}\text { Revolución ciudadana } \\
\text { Larga y triste noche neoliberal } \\
\text { Hacia el Buen vivir } \\
\text { Prohibido olvidar } \\
\text { Patria Grande } \\
\text { La prensa es mala }\end{array}$ \\
\hline $\begin{array}{l}\text { Lenin } \\
\text { Moreno }\end{array}$ & 2017 & $\begin{array}{l}\text { Revolución ciudadana } \\
\text { Vamos a dialogar } \\
\text { Plan toda una vida: Misión ternura } \\
\text { Plan toda una vida: Impulso joven } \\
\text { Plan toda una vida: Mis mejores años }\end{array}$ \\
\hline
\end{tabular}

Nota. Elaborado a partir de los discursos presidenciales $(2007 ; 2009 ; 2013 ; 2017)$. 


\section{Líneas argumentales destacadas} del discurso de toma de poder

En este estudio se consideran a las líneas argumentales como las evidencias que permiten un análisis objetivo del discurso, logrando un acercamiento multidisciplinario en relación con las estructuras semánticas, compuestas de complejidad léxica, uso de superlativos y tendencias ideológicas de los políticos en cuestión. El discurso en el acto de posesión como la promesa publica que el presidente hace a la nación, es a su vez, el horizonte de su gestión gubernamental (Tabla 3).
Las unidades de análisis fueron los cuatro discursos de R.C. y L.M., de los cuales, se extrajeron algunas oraciones en las que se usaron palabras de manera reiterativa, para una mejor visualización de las similitudes y diferencias, esto como parte del análisis comparativo establecido en la metodología (Tabla 4).

El análisis de carácter argumental toma como referencia el Análisis del Discurso propuesto por Teun Van Dijk (2016), estudia la estructura semántica compuesta de complejidad léxica, uso de superlativos y tendencias ideológicas.

\section{Tabla 4}

Frases relevantes de los discursos presidenciales de Rafael Correa (2007, 2009, 2013) y de Lenin Moreno (2017)

\section{Campaña Año Argumentos}

No negociaré con nadie la dignidad de la Patria. La Patria ya no está en venta.

El segundo eje de La Revolución Ciudadana fue y es la lucha contra la corrupción, mal enraizado en nuestra sociedad, pero también exacerbado por modelos, políticas y

2007 doctrinas que ensalzaron el egoísmo, la competencia y la avaricia como el motor del desarrollo social.

La lucha por una Revolución Ciudadana, consistente en el cambio radical, profundo y rápido del sistema político, económico y social vigente, sistema perverso que ha destruido nuestra democracia, nuestra economía y nuestra sociedad.

Rafael

Correa
Estamos de fiesta porque la Patria está renaciendo del caos mercantilista; ha sido arrebatada del baratillo privatizador.

El segundo eje de acción, la lucha contra la corrupción ha sido y es una práctica permanente y hasta obsesiva de nuestro gobierno. El único caso de corrupción en las 2009 altas esferas fue investigado y denunciado por nosotros mismos; y, por ello, gozamos de la credibilidad del pueblo.

Hemos concluido la primera etapa de nuestra Revolución Ciudadana, y la hemos concluido en paz, en medio de la mayoritaria aprobación de nuestro pueblo, cuya sagacidad siempre es mayor que la de los sesudos politólogos de ocasión. 
Continuación de Tabla 4.

\begin{tabular}{|c|c|c|}
\hline Campaña & Año & Argumentos \\
\hline \multirow{3}{*}{$\begin{array}{l}\text { Rafael } \\
\text { Correa }\end{array}$} & \multirow{3}{*}{2013} & $\begin{array}{l}\text { La Patria Grande, ya no es sólo un ideal de nuestros libertadores, es una necesidad de } \\
\text { supervivencia, un escudo contra la explotación, contra el neocolonialismo. }\end{array}$ \\
\hline & & $\begin{array}{l}\text { Se definen como contrapoder, es decir, actores políticos, pero no toleran respuestas } \\
\text { políticas. Si la prensa difama, desinforma, calumnia a nuestros gobiernos, es libertad de } \\
\text { expresión. Si algún presidente les responde, es atentado a la libertad de expresión; o sea, } \\
\text { unos son más libres que otros. }\end{array}$ \\
\hline & & $\begin{array}{l}\text { Esta es la razón de nuestra Revolución Ciudadana, aquí nadie está por ambiciones } \\
\text { personales, por ocupar un cargo, por pasar a la historia, estamos por servir, cambiar esas } \\
\text { relaciones de poder en función de las grandes mayorías, que aquí no manden pequeñas } \\
\text { élites, peores poderes extranjeros, si no que mande el pueblo ecuatoriano. }\end{array}$ \\
\hline \multirow{3}{*}{$\begin{array}{l}\text { Lenin } \\
\text { Moreno }\end{array}$} & \multirow{3}{*}{2017} & $\begin{array}{l}\text { Al diálogo todos llegaremos con un espíritu libre, con mente abierta, sin ideas } \\
\text { preconcebidas, dispuestos a ceder. Porque de eso se trata el diálogo. }\end{array}$ \\
\hline & & $\begin{array}{l}\text { En lo que respecta a la corrupción, nuestra lucha será implacable. ¡Vamos a practicarle } \\
\text { una cirugía mayor a la corrupción! Cuando empezamos a creer que el "yo" importa más } \\
\text { que el "tú", ya estamos entrando en el escabroso ámbito de la corrupción. }\end{array}$ \\
\hline & & $\begin{array}{l}\text { Este proceso también tiene una leyenda: la de la Revolución Ciudadana. Algún día } \\
\text { podremos narrar con orgullo a nuestros hijos y nietos, que fuimos testigos presenciales } \\
\text { de esa leyenda. Más aún: que con tesón fuimos parte de ella. }\end{array}$ \\
\hline
\end{tabular}

Nota. Elaborado a partir de los discursos de posesión $(2007 ; 2009 ; 2013 ; 2017)$.

El objetivo supremo es identificar y contrastar dos niveles de análisis de tipo argumental: primero, el compromiso que adquieren los candidatos por las ofertas que realizan a los electores; y el segundo, las obligaciones con sus potenciales votantes considerando los tres momentos antes comentados.

\section{Marco teórico}

Adriana Bolívar (2007) se refiere al discurso como interacción social, cognición, historia, diálogo y acción, porque en torno al discurso es posible crear significados sociales, construir conocimientos, contextualizar el presente con el pasado, relacionarse interpersonalmente $\mathrm{y}$, sobre todo, transformar realidades. Estas definiciones permiten identificar en el discurso un ejercicio holístico de comunicación, que permite ser estudiado a través del lenguaje ya sea oral o escrito.

El uso del lenguaje o "uso lingüístico" como lo denomina Fairclough (2008) en o para el discurso, es dinámico y se adapta con facilidad a quien haga uso de él, sin embargo, no siempre se utiliza de forma adecuada, y tampoco se transmite con elementos que aseguren una comunicación eficaz. La comunicación ha favorecido los cambios sociales dados en la 
historia, debido a la capacidad semiótica propia de los seres humanos. El discurso político se emplea para la transmisión de ideologías; es además el medio por el cual, los candidatos o gobernantes intentarán llegar y permanecer en el poder; "los mecanismos de persuasión entran en juego, adueñándose del discurso político" (Soler, 2011, p. 2).

El discurso político, además, requiere de ciertas habilidades al momento de ser pronunciado, "un orador poderoso puede controlar, en gran parte, el pensamiento de sus receptores" (Escandel, 1996, citado por Soler, 2011, p. 3). Si se consigue que el pueblo reciba un mensaje, claro, preciso y suficientemente estructurado y vinculado al contexto sociopolítico, quienes lo emitan, podrían asegurar su influencia directa en la mente de los receptores, "cerrar el círculo del discurso-poder, significa que aquellos grupos que controlan los discursos más influyentes tienen también más posibilidades de controlar las mentes y las acciones de los otros" (Van Dijk, 2016, p. 26).

Entra en juego entonces, el análisis del discurso; Teun Van Dijk (2016), se refiere además al análisis crítico, como un método que analiza las dinámicas del poder, es decir, es:

...un tipo de investigación analítica sobre el discurso que estudia primariamente el modo en que el abuso del poder social, el dominio y la desigualdad son practicados, reproducidos, y ocasionalmente combatidos, por los textos y el habla en el contexto social y político (p. 23).
De acuerdo con Adriana Bolívar (2007), para realizar un análisis crítico del discurso ACD es importante considerar una "definición de discurso como práctica social" ya que "ofrecen explicaciones en las que se repiten palabras clave como: poder, control, acceso, ideología, legitimidad, abuso de poder, ética (s)” (p. $31)$.

Existen naciones marcadas por conflictos políticos, mismos que han quedado en la historia, y que los actores políticos de la actualidad utilizan de acuerdo con el público al que se dirigen; "en los conflictos políticos, cada parte ofrecerá por una parte una retórica más sofisticada para públicos informados o especializados $\mathrm{y}$, por otra parte, un discurso simplificado ante los medios de comunicación" (Lange, 1993, citado por García, 2004, p. 63).

Ecuador ha pasado por distintos procesos, entre dictaduras y regímenes militares hasta 1979, en el cual un triunvirato militar, dio paso a la instauración de la democracia (Negrete, 1983), que se han referido antes como dictaduras blandas. Finalizando la década de los noventa y a inicios del nuevo siglo, se desencadena una serie de manifestaciones populares, que lograron destituir tres presidentes democráticamente electos, a saber, Abdalá Bucaram (1997), Jamil Mahuad (2000) y Lucio Gutiérrez (2005) (Morales, 2012).

Ahora bien, con respecto a la coherencia entre lo que se dice y se hace, está ligado a la ética personal de quienes aspiren generar cambios estructurales en el sistema administrativo del pueblo. Pedro Santander (2011) justifica el análisis del discurso, dado que en el lenguaje existe una 
capacidad de ocultar elementos, "solo conocemos la acción que se realiza con el lenguaje, la que podemos examinar y analizar empíricamente, en cambio, la intención detrás del autor queda oculta” (p. 211).

Podemos decir que el habla politica produce un cortocircuito en el orden simbólico que regula una comunidad o, en términos de Ranciére, en el reparto de lo sensible, es decir, se define como la irrupción de una palabra que plantea una nueva distribución $y$ articulación de los cuerpos y de los actos enunciativos; un desajuste entre las expectativas de una competencia y la actualización de una performance. Fundamentar esta idea-fuerza y observar sus derivaciones requiere seguir, aunque sea de manera parcial y asistemática, un recorrido argumentativo que atienda a la definición misma de lo político y a tópicos que conciernen a la enunciación en general y al habla política en particular (Bermúdez y Choi, 2014, p. 101).

A manera de antecedentes, además de la referencia principal, es decir libros y artículos de Teun Van Dijk, en Ecuador existen investigaciones pioneras en relación con las construcciones discursivas del expresidente Rafael Correa, por ejemplo, los estudios realizados por Esperanza Morales (2012), María Isabel Punín (2014), y Matthieu Le Quang (2018), que, en conjunto, exponen ampliamente el proceso que ha llevado el partido político de la Revolución Ciudadana, entre otros.

\section{Metodología}

En relación con el tema y la metodología de este estudio, se ha tomado como principal referencia los textos de Teun Van Dijk, que son Las Estructuras Ideológicas del Discurso en Ideología y Discurso (2003), Discurso y poder (2009), Análisis crítico del discurso (2016) y el Análisis crítico del discurso social y politico (1999); en este último el autor manifiesta:

El discurso político es aquello que es dicho por sus actores o autores, los politicos. Sin embargo, los políticos no son los únicos participantes en el terreno de la política. Se debería incluir también a los diversos receptores de sucesos comunicativos políticos, tales como la gente, el pueblo, los ciudadanos, "las masas" y otros grupos y categorías. La delimitación del discurso politico por sus principales "autores" es insuficiente $y$ necesita extenderse a un cuadro más complejo que incluya a todos sus participantes pertinentes, estén o no involucrados activamente en el discurso politico, o sean meramente receptores unidireccionales de la comunicación (Van Dijky Mendizábal, 1999, p. 13).

El análisis crítico del discurso permite analizar las estrategias que se han utilizado ya sea en palabras escritas o pronunciadas, para generar la relación contextual en las que son transmitidas. "Los

Global Media Journal México, 18(34), 70-93, enero - junio 2021. 
investigadores críticos no se contentan con ser conscientes de la implicación social de su actividad (como cualquier sociólogo de la ciencia lo sería), sino que asumen posiciones explícitas en los asuntos y combates sociales y políticos" (Van Dijk, 2016, p. 24).

Una vez establecida la metodología a seguir, se delimitó la muestra, para ello, se organizaron dos niveles para analizar: tres principales mensajes distribuidos en la campaña presidencial, promovida por R. C. en los años 2006, 2009, 2013 y por L. M. en 2017, dando un total de 12 eslóganes (Tabla 1). De los planes de gobierno presentados al Consejo Nacional Electoral (CNE), se extrajeron 38 elementos entre palabras y frases cortas (Tabla 2), y de los cuatro discursos de posesión presidencial en los años que le correspondió a cada uno de estos personajes, se extrajeron 29 elementos, también entre palabras y frases cortas como "gente de manos limpias y corazones ardientes" (Tabla 3).

Se distribuyeron las palabras o frases mencionadas en tablas comparativas (Tablas 1, 2 y 3), logrando definirse como conceptos básicos o puntos principales y en gráficos para el posterior análisis comparativo. Debido a la extensión de las palabras y por cuestión de orden, se tomaron los tres momentos de acuerdo con los personajes analizados y con los años en que se distribuyeron los mensajes. Luego de esta separación, se realizaron figuras que muestran si hubo relación o no, de las palabras dichas en los tres momentos examinados.

\section{Resultados}

Los mensajes para las campañas políticas se transmitieron en diferentes escenarios, combinados entre vallas publicitarias, grafitis, volantes, publicidad en radio, televisión, prensa y plataformas digitales, usados en los tres periodos de R.C. de 2006-2009 y 2013, en igual proporción con respecto al 2017 para la campaña de L.M.

\section{Primer nivel, compromiso con posibles electores}

En este primer nivel también se extraen los conceptos básicos plasmados en el Plan de Gobierno de ambos personajes; en el caso de R.C. los años 2006-2009 y 2013 (cabe mencionar que el del 2009 no se ha publicado en la web, por lo que no fue posible analizarlo) y del presidente L.M. en el 2017

Cada vez que existe una convocatoria a elecciones presidenciales, el Plan de Gobierno de los partidos y movimientos políticos con su respectivo candidato a la presidencia de la República, son presentados al Consejo Nacional Electoral (CNE), y se usan como documentación de análisis prioritario.

\section{Segundo nivel, compromiso con los potenciales votantes}

Este nivel integra los conceptos utilizados en los discursos de toma de poder una vez ganadas las elecciones presidenciales, es así como consecutivamente en el 2007, 2009 y 2013, R.C. subió al púlpito para dar su primer mensaje a la 
Nación; y en el año 2017, L. M. es electo a la Presidencia de la República, quien hasta ese momento continúa con la línea política de su antecesor.

\section{Unidades de análisis}

A continuación, se encuentran las figuras con las tres frases o eslóganes de campaña, las palabras o frases extraídas de los Planes de Gobierno y del discurso de posesión de cada año. De ese modo, la Figura 1 analiza el 2007, la Figura 2 el 2009, la Figura 3 el 2013, y la Figura 4 el 2017. Luego, se realiza el análisis e interpretación de estas.

Como se puede observar, las palabras o conceptos básicos utilizados por R.C. en las frases de campaña del año 2006 (Figura 1), son simples. Por ejemplo, la frase, Dale Correa, fue utilizada luego de que un simpatizante sacara su correa (léase cinturón) y se la otorgó al precandidato presidencial Rafael Correa, en símbolo de que con ese elemento castigaría a los políticos corruptos, "Dale correa, le dijo" (Diario El Universo, 2007). Entonces, se utilizó para la campaña del 2006, de hecho, se convirtió en un eslogan publicitario. No fue utilizada en el Plan de Gobierno de ese año, pero la expresión coloquial dale fue mezclada con frases como dale trabajo, dale vivienda, dale educación, entre otras; y se asoció como parte de la retórica inicial. En el discurso de posesión del 2007, esta expresión desaparece; en campaña fue una frase populista, útil para sus potenciales votantes, pero ya en el espacio formal, en la toma de poder, necesita dirigirse directamente a quienes por primera vez lo eligieron mandatario.

\section{Figura 1}

Frases utilizadas por Rafael Correa en campaña, Plan de Gobierno y discurso de posesión (2006-2007)

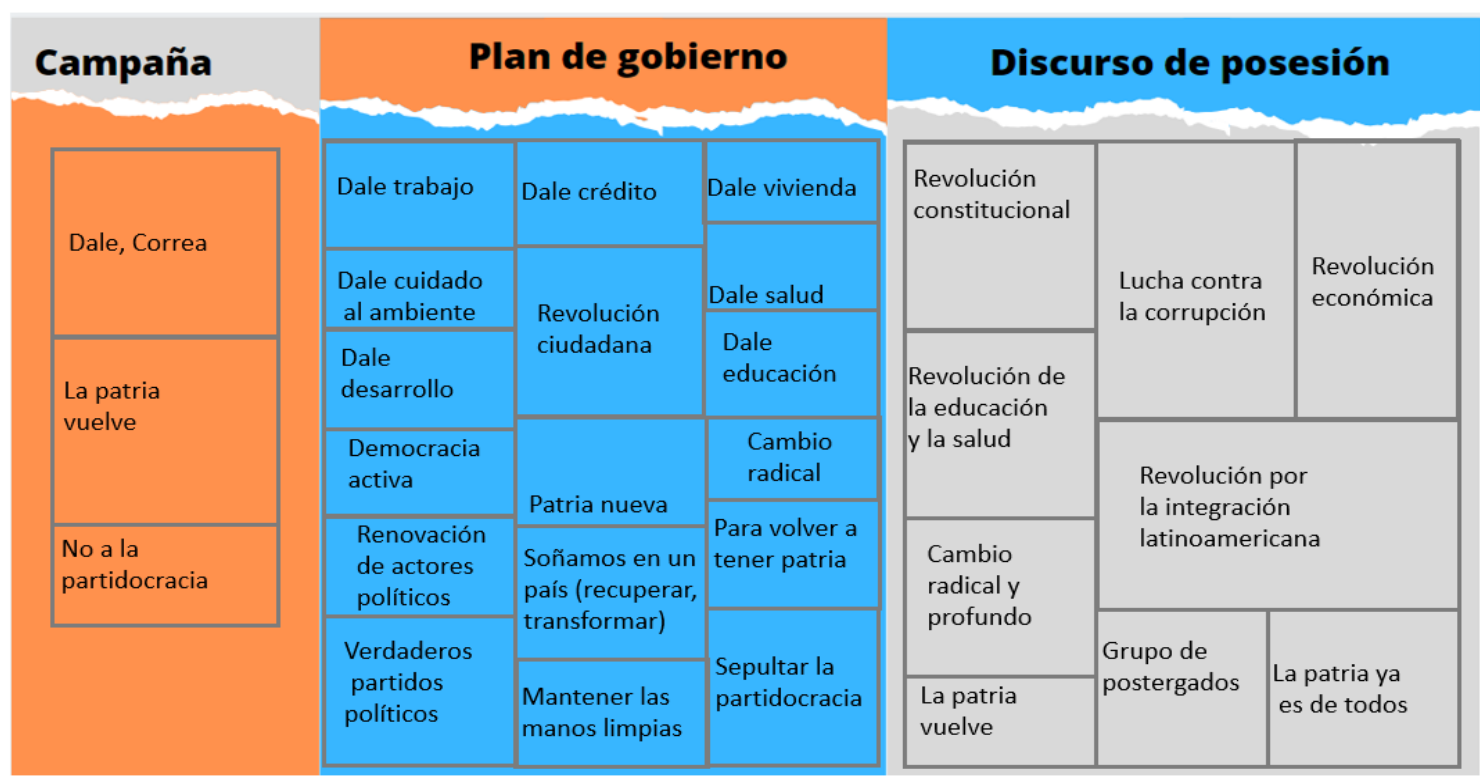

Nota. Elaborado a partir del discurso de posesión (2007). 
R.C. no utiliza superlativos en ninguno de los tres momentos, sin embargo, emplea la repetición de conceptos, como una acción clave para posicionar su nombre y su proyecto político en la mente del electorado.

Podemos esperar operaciones de repetición en el nivel de los sonidos (aliteraciones y rimas), formas de oración (paralelismos) y significando (repetición semántica), como una de las estrategias importantes que merecen atención respecto de significados preferidos y para mejorar la construcción de significados en modelos mentales y su memorización en intentos de procesos de persuasión o luego de recordación (Van Dijk y Mendizábal, 1999, p. 59).

La repetición de ciertas palabras (Figura 1) responde a una estrategia bien utilizada en la primera campaña presidencial, no consiste en repetir la frase, sino en relacionar una palabra y construir otra frase, por ejemplo, dale correa, o también revolución ciudadana que en este caso es mencionada en el Plan de Gobierno y en el discurso de posesión presidencial de Rafael Correa del año 2007. Además, en este último, la palabra revolución se inserta dentro de los cinco ejes de acción:

1. Revolución constitucional.

2. Revolución contra la corrupción.

3. Revolución económica.

4. Revolución de la educación y la salud.

5. Revolución por la integración

latinoamericana.
En el año 2006, R. C. al inicio de la campaña política, seguido de su Plan de Gobierno y discurso de posesión presidencial, deja marcada su ideología política, que aparentemente se alejaba de las ya existentes en el país, llamando a los otros partidos políticos la partidocracia, palabra que utiliza en los tres momentos de análisis, además, usa otras expresiones como "renovación de actores políticos", “cabría preguntarse qué porcentaje de los caciques de los partidos políticos se ha renovado y a quién están representando esos diputados si a sus mandantes o a sus caciques" (Correa, 2007). Al respecto Teun Van Dijk (1999) menciona:

Las evaluaciones son caracteristicamente polarizadas: mientras NOSOTROS somos democráticos, ELLOS no lo son y en tanto NUESTROS soldados, o aquellos quienes comparten nuestra causa, son combatientes de la libertad, los de los OTROS son obviamente terroristas (Chomsky, 1985; 1987; Van Dijk, 1995a). Lo mismo se puede afirmar respecto a nuestras politicas $y$ decisiones politicas, que invariablemente beneficiarán al país y a todos los ciudadanos, mientras los de los otros no ( $p$. 44).

Sin embargo, a pesar de que la destrucción de la llamada partidocracia fue una de las principales promesas anunciadas en la campaña, plan de gobierno y discurso de posesión para el periodo 2007- 2011. En el año 2009, se convocó a elecciones seccionales; el Diario El Universo (2009), iniciaba 
una noticia así: "Decenas de candidatos a alcaldes, prefectos y asambleístas inscritos en el movimiento gobiernista PAIS son de la criticada 'partidocracia'".

Otra palabra utilizada en los tres momentos es Patria, sin embargo, hay ciertas variaciones en la relación semántica y en la lógica discursiva, es así como en la campaña usa la frase "la Patria vuelve", y en el Plan de Gobierno menciona "para volver a tener Patria".

Es evidente una contradicción, menciona que su movimiento Alianza PAÍS "sueña con una Patria nueva", con "un Ecuador diferente". Ecuador para entonces estaba conformado de 14.06 millones de personas, y es necesario pensar en la historia cognitiva, cultural, social, en fin, no se puede pretender desechar aspectos originarios y de la noche a la mañana construirlos diferentes.

Luego en el discurso de posesión menciona que "la Patria ya es de todos" y que es "altiva y soberana", dejando la posibilidad de discutir aquello de una patria nueva mencionada en el Plan de Gobierno que dirigió a la ciudadanía electora, debido a que apenas iniciaba su mandato y para entonces aún no existían cambios en ella.

Ante el posicionamiento de la sencilla palabra Patria, dos años después, para la campaña de reelección, regresa a este concepto (Figura 2), relacionándolo con Dale Patria y se insiste en la frase Patria altiva y soberana utilizada en el primer discurso de posesión del 2007 por R.C. En su segundo discurso del 2009, se recupera el concepto de Patria Nueva, en este caso hay dos frases que se repiten, pero toman un nuevo matiz, de tal modo que aparece otro concepto mencionado por R.C. en sus discursos y es Vende Patria.

Un día, casi no se podrá creer que existiera un proceso de monumental estafa que se hubiera podido rodear de un halo de respetabilidad, de secretismo "técnico"; que nadie, durante décadas, hubiera sido capaz de destapar esta olla de grillos; que, legiones de tecnócratas, vende patrias, hayan podido vivir y lucrar del cuento de la deuda, en contra de su pueblo (Correa, 2009).

Como se puede observar, este nuevo término es utilizado para hablar contra la oposición que para entonces había crecido, aunque R. C. aún gozaba de una alta simpatía ciudadana.

En estos nuevos comicios, la oposición, muy dividida, centró todos sus esfuerzos en lograr una segunda vuelta, pero ha quedado muy lejos de lograrlo. El segundo puesto del expresidente Lucio Gutiérrez no es muy alentador debido, sobre todo, a que no ha conseguido llegar ni al 30\% de los votos, es decir, a 21 puntos de Correa (Diario El País, 2009). 


\section{Figura 2}

Frases utilizadas por Rafael Correa en campaña, Plan de Gobierno y discurso de posesión 2009



Nota. Elaborado a partir del discurso de posesión (2009).

A este punto de su presidencia y reelección, R.C. se mantiene en la ideología planteada al inicio de su carrera política, es así como, tanto en la campaña del 2009 como en el nuevo discurso de posesión, recalca el término Revolución Ciudadana, añadiendo que "está en marcha" y que "es irreversible"; para entonces amplía el concepto y plantea poner en marcha el Socialismo del siglo XXI, implementado en los países latinoamericanos como Venezuela, Ecuador, Bolivia, Brasil y Argentina.

\section{El perfil político de Correa llamó la atención} de los medios el año 2005, cuando desempeñaba sus funciones como ministro de Economía en el gobierno de Alfredo Palacio (2005). Desde aquel tiempo ya se evidenció un acercamiento con Venezuela. Otra de las acciones que perfilaron una marcada posición de lo que se llamó la nueva izquierda, que ha sido promocionada como el Socialismo del Siglo XXI (Punín y Rúas, 2015, pp. 84-85).

Así pues, R.C. se mantiene en la estrategia original; utiliza el distanciamiento de los otros partidos políticos tradicionales del país, refiriéndose a sus aliados como "gente de manos limpias y corazones ardientes", mientras los demás continúan sumergidos en "una larga y triste noche neoliberal" (Correa, 2009).

Las ideologías surgen generalmente cuando dos o más grupos tienen intereses conflictivos entre sí, cuando aparecen la lucha social o la competencia en una situación de dominio. Tanto en el plano 
cognitivo como en el discursivo, esta oposición se manifiesta a través de diversas formas de polarización, como en el caso de los pronombres Nosotros y Ellos. Ya hemos visto que la estrategia general del discurso ideológico es poner énfasis en Nuestros aspectos positivos y Sus aspectos negativos, una forma de polarización que se aplica semánticamente por contraste (Van Dijk, 2003, p. 6).

En este mismo orden de ideas, R.C., identifica un nuevo grupo opositor y lo conceptualiza como prensa corrupta. "Felizmente, pese a todas esas falacias aupadas muchas veces por una prensa corrupta, nuestra nueva Constitución ya es una realidad, y la piedra fundacional del cambio de época, de este nuevo tiempo de la Patria" (Correa, 2009). De acuerdo con este contexto, es posible concluir que, para el primer mensaje a la nación, en este nuevo periodo presidencial, R.C. concentra su discurso en insistir que su proyecto Revolución Ciudadana no será detenido por los grupos opositores que previamente él ha identificado.

\section{Figura 3}

Frases utilizadas por Rafael Correa en campaña, Plan de Gobierno y discurso de posesión 2013

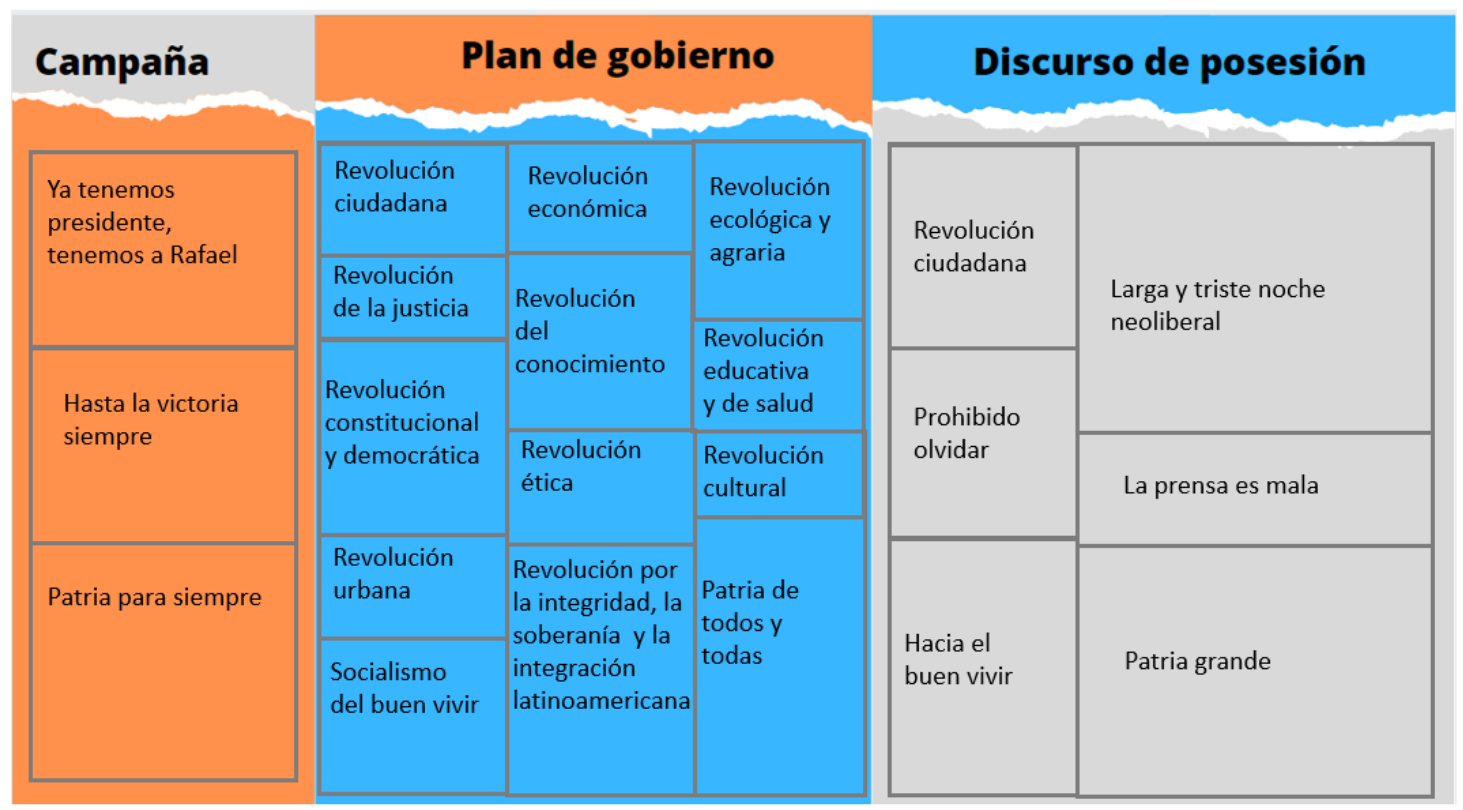

Nota. Elaborado a partir del discurso de posesión (2013).

En este nivel de análisis de las expresiones que utilizó R.C. para las campañas electorales, los Planes de Gobierno (2007 y 2013), y en los tres discursos de posesión presidencial, es posible interpretar que una estrategia para conectar con la ciudadanía es el uso de una complejidad léxica sencilla con frases populares. Para la campaña presidencial del 2013 (Figura 3), decide utilizar el eslogan Ya tenemos 
presidente, tenemos a Rafael, en un intento de imponer una decisión del pueblo, para que ni siquiera piense en otra opción, la Patria para siempre y Hasta

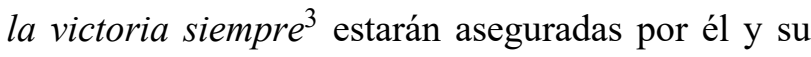
movimiento político, que más tarde se tradujeron en una votación masiva.

En el transcurso de este análisis ha sido evidente que R.C. no utiliza superlativos, la estrategia está en utilizar frases como La Revolución Ciudadana y que a la vez estas son combinadas con otros elementos, es así que para el Plan de Gobierno del 2013, amplía los ejes de la Revolución, donde de cinco pasan a ser once, dando fuerza a la Revolución ecológica y la Revolución cultural, a través de otras expresiones como "El socialismo del Buen vivir y Prohibido olvidar" mencionadas en el discurso de posesión para el tercer periodo de presidencia 20132017.

Esta ideología se mantiene lejos del resto de los partidos políticos, es adepto al Socialismo del siglo XXI, e insiste en mencionar que Ecuador antes de su llegada vivió una "larga y triste noche neoliberal"4. Para reforzar esta idea, en su tercer discurso de posesión plantea otro concepto, "prohibido olvidar", es decir, desde su perspectiva, el pueblo debe recordar que, a su llegada, la Patria grande ha cambiado, sin embargo, para entonces, también había crecido la oposición, aunque la popularidad de R.C. le permitía continuar en primer lugar.

${ }^{3}$ Como se menciona en el spot publicitario "Bicicleta" de Rafael Correa: https://youtu.be/0fZgCG2N5KM
De esta manera, tras 7 años de gobierno, Correa se mantendrá en el mandato hasta el 2017 y así se convertirá en el presidente que más tiempo ha durado en el cargo en las últimas décadas. Los tres mandatarios que lo precedieron no pudieron terminar su periodo presidencial, que en Ecuador es de cuatro años (Diario El Comercio, 2013).

Correa relaciona a la oposición directamente con la prensa, insistiendo en su tercera ascensión, que la prensa es mala, su polarización con los medios de comunicación privados del país radica en considerar que estos a más de no cumplir con la obligación de informar verazmente a la ciudadanía, le han dado rienda libre a desprestigiarlo.

Estoy persuadido de que una prensa libre es vital para la democracia, pero estoy igualmente convencido de que una mala prensa es mortal para esa misma democracia. Y en Latinoamérica, no solo en Ecuador, en Latinoamérica, con las honrosas excepciones de siempre, la prensa es mala, muy mala (Correa, 2013).

Teun Van Dijk (2009), se refiere al uso de términos negativos como una "reproducción de los prejuicios" hacia los demás:

\footnotetext{
4 "Larga y triste noche neoliberal" frase utilizada en el discurso de posesión del año 2013, por Rafael Correa y replicada masivamente en las redes sociales.
} 
...el abuso de poder solo puede manifestarse

en el uso del lenguaje si existe la posibilidad de variación o elección, tal como decir de una misma persona que es un terrorista o un luchador por la libertad, según la posición o la ideología del que habla (p. 24).

Si bien R.C. hace referencia en contra de la prensa, él mismo utilizaba los mecanismos de reproducción discursiva, pues tenía en su poder los medios públicos, a los que les dio protagonismo a partir de la Ley Orgánica de Comunicación (LOC-2013), aprobada el 14 de junio de 2013, articulado legal que aprobó con la oposición mayoritaria de los propietarios de los medios de comunicación, por lo cual se cumplió con parte de las promesas de campaña.

Si el discurso controla las mentes y las mentes controlan la acción, para quienes ocupan el poder controlar el discurso es absolutamente esencial. ¿Cómo lo logran? Si los eventos comunicativos no solo están compuestos por el texto u la conversación "verbales" sino también por un contexto que influye en el discurso, el primer paso que debe darse para controlar el discurso es controlar sus contextos. Por ejemplo, las élites $u$ organizaciones poderosas pueden decidir quiénes, cuándo, dónde y con qué objetivos participan de un determinado evento comunicativo. Esto significa que debemos examinar en detalle los modos en que quienes están en el poder regulan el acceso al discurso, como se observa típicamente en el caso de una de las formas más influyentes de discurso público, la de los medios de comunicación masiva (Van Dijk, 2009, p. 31).

El 4 de abril de 2017, el Consejo Nacional Electoral (CNE) declaraba a Lenin Moreno, como ganador de la segunda vuelta de las elecciones a la presidencia de la República del Ecuador (BBC Mundo, 2017). El candidato propuesto por el movimiento político Alianza PAÍS, llegaba al poder a partir de la misma línea ideológica, su campaña electoral deja atrás las frases compuestas con las palabras que utilizaba R.C. y se toma como bandera las frases "Vamos por más", "Unidos somos invencibles" y "El futuro no se detiene". Es posible identificar que esta última va ligada al proyecto Revolución Ciudadana en el Plan de Gobierno del 2017 (Figura 4).

La propuesta pasa de cinco a doce ejes de la revolución; además, en este plan regresa al concepto de Patria grande, pero distorsiona el Socialismo del siglo XXI, pasando a ser Socialismo del Buen vivir.

De igual modo, en las palabras que utilizó L.M. hay poca complejidad léxica, a diferencia de R.C. que repetía conceptos bien definidos, en este caso es casi imposible predecir lo que continúa, pues no define palabras ni las repite, tan solo la palabra diálogo se roba el protagonismo, y se planteó como uno de sus conceptos básicos. 


\section{Figura 4}

Frases utilizadas por L.M. en campaña, Plan de Gobierno y discurso de posesión de 2017

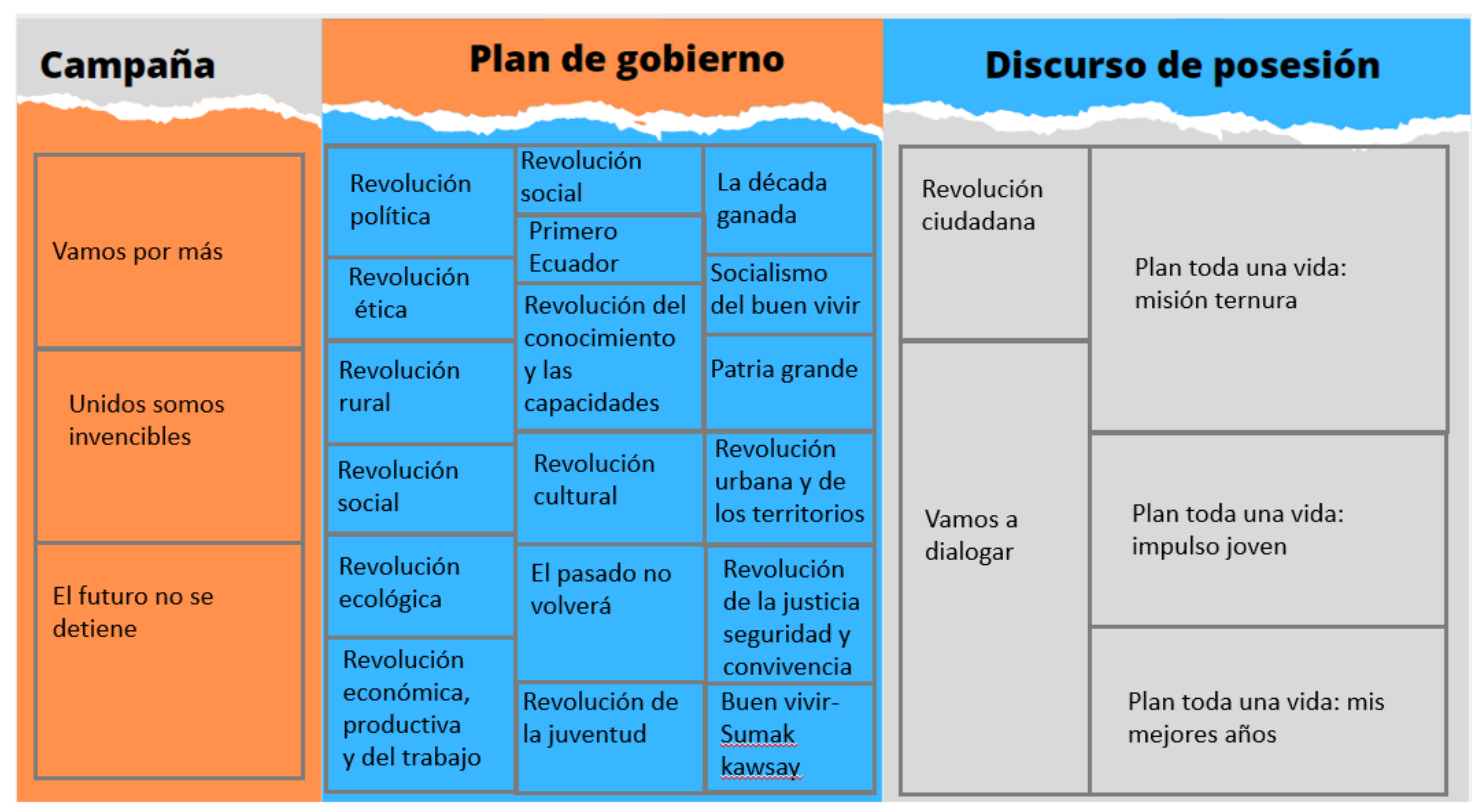

Nota. Elaborado a partir del discurso de posesión (2017).

En su discurso de posesión, L.M., utiliza el superlativo excelentísimo, pero este se encuentra dentro de una historia que narra con la finalidad de ilustrar que en su gobierno no perseguirá a quienes se rían de él.

- Señor presidente, ¿podemos presentar esta obra en la cual la gente se ríe de usted?

- El presidente le contestó: La República no se perderá porque el pueblo se ría de un gobernante; se perderá cuando el gobernante se ría de su pueblo (Moreno, 2017).

Como ya se ha indicado con respecto a la ideología de L.M. hasta el momento de su ascensión, se consideraba parte del proyecto Revolución Ciudadana, pero más adelante, marca el distanciamiento absoluto y frente a la ciudadanía. R.C. deja de ser su amigo para ser un prófugo de la justicia, debido a diferentes casos de corrupción que salieron a la luz durante el gobierno de Lenin Moreno.

Sin diplomacias ni medias tintas, los discursos del presidente se han ido cargando de palabras densas para su predecesor, para el régimen de Nicolás Maduro y para prácticamente todo lo que esté ligado a la corriente de izquierdas que imperó en América Latina en la última década. El socialismo del siglo XXI pasó con Moreno de ser el programa ideológico con el que ganó las elecciones en mayo de 2017 a un modelo “perverso" y “corrupto”, como ha repetido en sus comunicados la presidencia de 
Ecuador. Lo único que aún persiste de aquellos dogmas es la promesa a las clases populares de que todo lo que se decide tiene la prioridad de protegerlas (España, 2019).
Hasta aquí el análisis y la interpretación del discurso en los tres momentos; a continuación, se ha separado por años las frases que más se han repetido o aquellas con las que más se identificaron los actores políticos en análisis a través de nubes de palabras (Figura 5).

\section{Figura 5}

Frases utilizadas por Rafael Correa y Lenin Moreno en campaña, Plan de Gobierno y discurso de posesión 2006-2007, 2009, 2013 y 2017

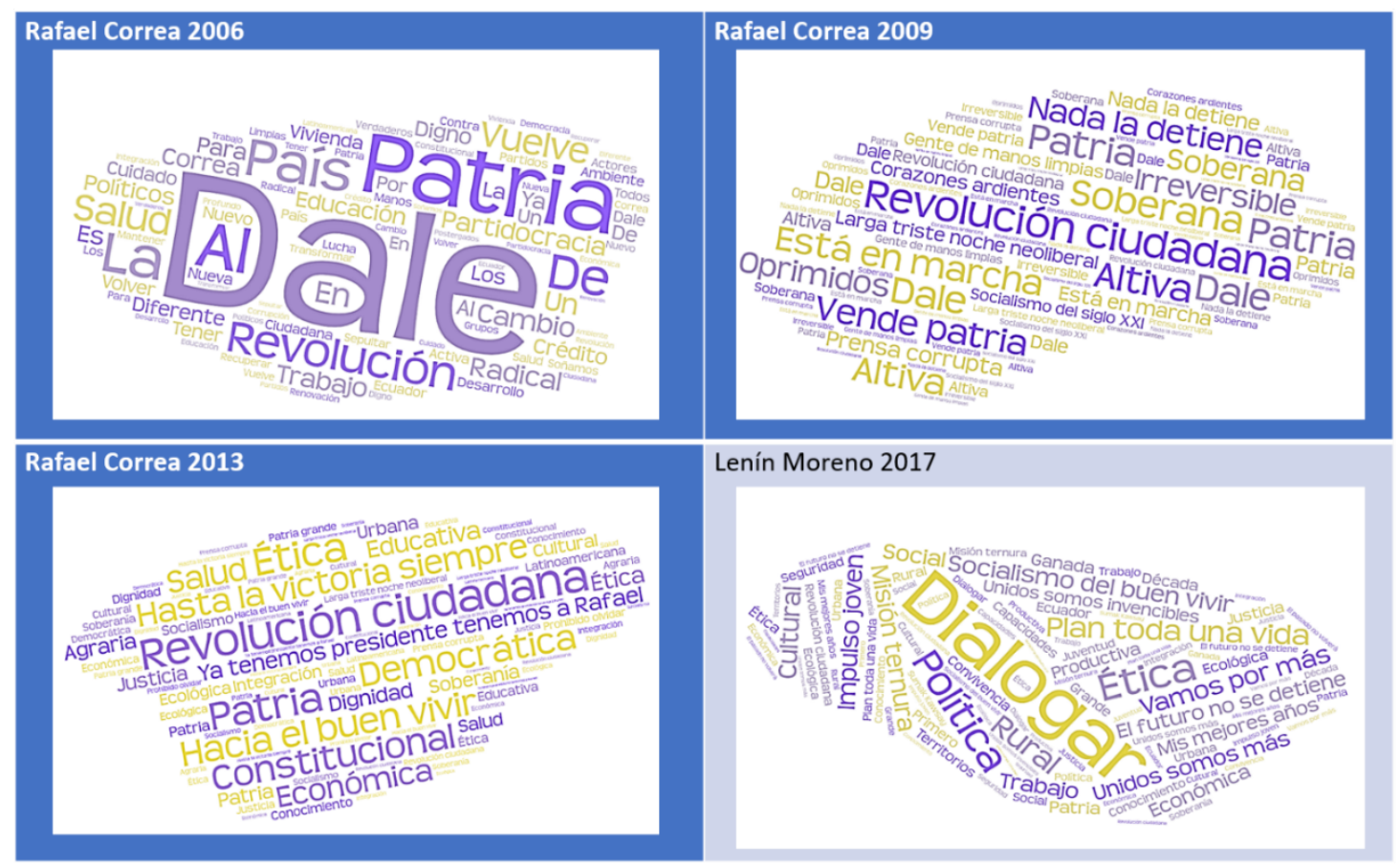

Nota. Elaborado a partir de los discursos de posesión presidencial citados en la Tabla 4.

Dada la diferencia de conceptos propuestos de Rafael Correa y Lenin Moreno, y a pesar de que este último perteneció por diez años al mismo movimiento e incluso, fue vicepresidente de la República los dos primeros periodos, es posible deducir que Moreno trata de persuadir a la ciudadanía, marcando el distanciamiento con sus coidearios, para evitar el compromiso de haber pertenecido al gobierno anterior, y deslindarse de responsabilidades sobre todo en el tema económico y social.

De hecho, las ideologías organizan a la gente y a la sociedad en términos polarizados. La pertenencia a un grupo tiene que ver ante todo con quién pertenece o no al grupo y cómo nos distinguimos de los demás gracias a las acciones, objetivos, 
normas y recursos del grupo (Van Dijk, 2003, p. 2).

Para ello, en el discurso pronunciado después de su posesión, L.M. toma el papel de protector, revelando que su gobierno no podrá trabajar, debido a los desfalcos económicos que se dieron durante el gobierno de R.C. A pesar de ello, L.M. todavía pertenece al movimiento político Alianza PAÍS, mientras que Correa y sus adeptos, el 8 de julio de 2020, crearon otro movimiento político, denominado Unión por la Esperanza (Unes).

\section{Conclusiones}

Este artículo identifica los principales elementos discursivos utilizados por los dos últimos gobiernos de Ecuador, el de Rafael Correa que se estudia desde el año 2007 hasta el 2017 y el de Lenin Moreno desde el 2017 hasta el 2021 ambos siendo parte del gran corpus de estudio, ordenados en tres momentos 1 . Campaña electoral, 2. Plan de gobierno y 3 . Toma del poder.

Rafael Correa en las campañas electorales de los tres periodos, logra posicionar su imagen a través de frases de complejidad léxica sencilla, pero usando la reiteración constante, destacando el concepto de la Revolución Ciudadana, asociada a frases cortas como: Patria nueva, Revolución Ciudadana está en marcha y nada la detiene, y Hasta la victoria siempre. Lo mismo hace en los Planes de Gobierno del 2006 y 2013, y en los discursos de posesión al mandato (Tabla 3 y 4 ).

En los tres momentos analizados, de los tres periodos presidenciales R.C. no hace uso de superlativos, sin embargo, la repetición de conceptos es evidente, aunque no repite frases textuales, utiliza ideas básicas y las mezcla con otros elementos. Durante una década en el poder logra posicionar su ideología política, separándose de las tradicionales del país (Figuras 1, 2, 3).

En relación con el segundo momento, Correa mantiene una estrategia, se apoya en la construcción de frases definidas, y los Planes de Gobierno (2007, 2009 y 2013) vuelve a tomarlos como bandera. Por el contrario, L.M. (2017), utiliza en la campaña electoral como en el Plan de Gobierno, conceptos posicionados por R.C, pero ya en el discurso de toma de mando no es clara su posición con respecto a ello, los cambios se dan sobre todo porque no es posible identificar claramente los conceptos que desea posicionar, explica que más allá de la persecución, él estaría abierto al diálogo.

En el tercer momento del análisis y el segundo nivel de compromiso que adquieren los presidentes con los potenciales votantes, el discurso de Rafael Correa mantiene la estructura discursiva que utiliza en campaña, y en Planes de Gobierno, se evidencia que, en el discurso de posesión del 2007, fue auténtico en la estructura. En cambio, en los discursos transmitidos en el 2009 y 2013, se centra en breves informes de gestión (rendición de cuentas), los ejes de la revolución ciudadana pasaron de ser cinco a trece, y para el último discurso identifica más grupos opositores.

Global Media Journal México, 18(34), 70-93, enero - junio 2021. 
No obstante, L.M no logra un análisis real de la situación en la que recibía el país y, por lo tanto, faltó un compromiso claro con los potenciales electores. Aunque no ha sido objetivo de esta investigación, es posible reconocer a través de los distintos mensajes que da a la nación, que Lenin Moreno tiene una limitación discursiva constante.

Se muestra una polarización marcada con respecto a la ideología, Moreno deja atrás la Revolución Ciudadana y el Socialismo del siglo XXI, a pesar de que sigue perteneciendo al movimiento político Alianza PAÍS. En palabras de Moreno hay poca complejidad léxica (Figura 4), aunque no hay un planteamiento definido en sus conceptos y no usa mayores reiteraciones.

Se ha identificado que los presidentes de la República del Ecuador, Rafael Correa y Lenin Moreno, fueron cambiando progresivamente el discurso público. El primero lo hizo para asegurar su permanencia en el poder durante los diez años. Mientras que el segundo, lo hace por el cambio de ideología política, que utiliza para justificar la situación en que recibió al país y las pocas acciones que ha llevado durante su mandato a finalizar en el 2021.

\section{Referencias bibliográficas}

Asamblea Nacional Constituyente (2018). Código de la Democracia. https://bit.ly/3gXUn40

BBC Mundo. (4 de abril de 2017). El oficialista Lenin Moreno es declarado vencedor de las elecciones presidenciales en Ecuador. BBC News Mundo. https://bbc.in $/ 3 \mathrm{fZi} 7 \mathrm{mF}$

Bermúdez, N., y Choi, D. (2014). Los modos de decir en la política. Una intervención al análisis del discurso. Revista Mexicana de Ciencias Políticas y Sociales, 59(221), 99-119. https://doi.org/10.1016/s01851918(14)70817-7

Bolívar, A. (2007). Análisis del discurso: por qué y para qué. Editorial CEC, S.A.

Chilton, P., y Shäffner, C. (2002). Politics as text and talk: Analytic approaches to political discourse. John Benjamins Company.

Consejo Nacional Electoral. (2006). Elecciones presidenciales 2006. https://bit.ly/3ySSc8U

Consejo Nacional Electoral. (2013). 2013 Elecciones Generales. https://bit.ly/3yY55ie

Consejo Nacional Electoral. (2006). Plan de Gobierno del Movimiento PAIS 2007-2011. https://bit.ly/3wYScTn Consejo Nacional Electoral. (2013). Plan de Gobierno del Movimiento PAIS 2013-2017. https://bit.ly/3jfVQEz

Consejo Nacional Electoral. (2017). Plan de Gobierno del Movimiento PAIS 2017-2021. https://bit.ly/3y2qrK1

Consejo Nacional Electoral. (2017). Resultados electorales 2017. https://bit.ly/2TuWD9H

Constante, S. (24 de marzo de 2017). Lenin Moreno se distancia de Correa en la recta final de la campaña. Diario El Pais. https://bit.ly/3fZNJsy

Global Media Journal México, 18(34), 70-93, enero - junio 2021. 
Discurso y poder en Ecuador: análisis del discurso de los presidentes...

Correa, R. (2007, 15 de enero). Discurso de posesión presidencial [Video]. Toma de posesión. https://www.youtube.com/watch?v=G122Rf_xdM

Correa, R. (2009, 10 de agosto). Discurso de posesión presidencial [Texto]. Toma de posesión. https://www.voltairenet.org/article161992.html

Correa, R. (2013, 10 de agosto). Discurso de posesión presidencial [Video]. Toma de posesión. https://www.youtube.com/watch?v=N0X9rDrO0ik\&app=desktop

Diario El Comercio. (18 de febrero de 2013). Los 7 triunfos de Correa en las urnas. https://bit.ly/3g3aaNu

Diario El Comercio. (28 de octubre de 2017). Contraloría notificó destitución a Jorge Glas, quien impugnará. https://bit.ly/3vHD4t4

Diario El País. (27 de abril de 2009). Rafael Correa, reelegido presidente de Ecuador en la primera vuelta. https://bit.ly/2SHhHJL

Diario El Universo. (15 de enero de 2007). Las correas que se fueron en la campaña. https://bit.ly/3ccHCQi

Diario El Universo. (29 de marzo de 2009). "Partidocracia", la base y eje del movimiento gobiernista. https://bit.ly/3ieamfp

España, S. (18 de marzo de 2019). Ecuador rompe hasta en lo simbólico con el modelo político de Rafael Correa. Diario El País. https://bit.ly/3p9V4tD

Fairclough, N. (2008). El análisis crítico del discurso y la mercantilización del discurso público: las universidades. Discurso y Sociedad, 2(1), 170-185. https://bit.ly/3p7WsNq

Fair, H. (2010). Una aproximación al pensamiento político de Michel Foucault. Polis, 6(1), 13-42. https://bit.ly/3dd2aIW

García, D. (2004). La metáfora en el discurso político. Reflexión, 83(2), 61-72. https://bit.ly/3c6m0oM

Le Quang, M. (2018). La Revolución Ciudadana en escala de grises: avances, continuidades y dilemas. IAEN.

Morales, E. (2012). El Discurso político de Rafael Correa (Presidente de Ecuador). Tonos Digital, $23,30$. https://bit.ly/2S0T6Q9

Moreno, L. (2017, 10 de agosto) Discurso de posesión presidencial [Video]. Toma de posesión. https://www.youtube.com/watch?v=8GnShGA9D3g\&feature=youtu.be

Movimiento Alianza PAIS. (2017). Sala de prensa. Alianza PAIS. https://bit.ly/3uGUvbU

Negrete, A. (1983). Ecuador: realidad y perspectivas de la democracia. En R. Sohel (Comp.), Los modelos de la crisis (pp. 205-218). El Conejo.

Organización de Estados Americanos. (2010). Informe de la misión de observación electoral para las elecciones generales de presidente y vicepresidente de la república, asambleístas nacionales, asambleístas provinciales, prefectos provinciales, alcaldes, concejales rurales, concejales urbanos, parlamentarios al parlamento andino y miembros de juntas parroquiales rurales celebradas en la república de Ecuador el 26 de abril y el 14 de junio de 2009. https://bit.ly/2TuXLKt

Global Media Journal México, 18(34), 70-93, enero - junio 2021. 
Punín, M. (2014). Una visión comunicacional de la política en Ecuador: Rafael Correa y su bicicleta. Obets, 9(2), 361-376. https://doi.org/10.14198/OBETS2014.9.2.05

Punín, M. y Rúas, J. (2015). La estrategia comunicacional y mediática de Rafael Correa. Redmarka: Revista de Marketing Aplicado, 08(014), 75-101. https://doi.org/10.17979/redma.2015.01.014.4882

RAE. (2001). Discurso. En Diccionario de la Lengua Española. https://bit.ly/34DRui0

Santander, P. (2011). Por qué y cómo hacer Análisis de Discurso. Cinta de Moebio, 41, $207-224$. https://doi.org/10.4067/s0717-554x2011000200006

Soler, R. (2011). El discurso político como marco de relaciones de poder asimétricas. Tejuelo: Didáctica de La Lengua y La Literatura, (11), 128-144. https://bit.ly/2S5nf0B

Van Dijk, T. A. (2003) Las estructuras ideológicas del discurso, en Ideología y Discurso. Ariel Lingüística, pp. $55-75$.

Van Dijk, T. (2005). Política, ideología y discurso. Quórum Académico, 2(2), 15-47. https://bit.ly/3pi0gM3

Van Dijk, T. (2009). Discurso y poder. Contribuciones a los estudios críticos del discurso. Editorial GEDISA.

Van Dijk, T. (2016). Análisis Crítico del Discurso. Revista Austral de Ciencias Sociales, 30, 203-222. https://doi.org/10.4206/rev.austral.cienc.soc.2016.n30-10

Van Dijk, T. y Mendizábal, I. (1999). Análisis del discurso social y político. Abya-Yala.

Wodak, R., y Menz, F. (1990). Sprache in der Politik - Politik in der Sprache: Analysen zum öffentlichen Sprachgebrauch. Drava.

Nota. Los eslóganes se han obtenido a través de un rastreo digital, realizado durante los meses de enero, febrero, marzo y abril del 2021 en las páginas: https://www.alianzapais.com.ec/; https://bit.ly/3jeeJrq; https://bit.ly/2TZhEcV; entre otras.

Global Media Journal México, 18(34), 70-93, enero - junio 2021. 